\title{
Effect of Reduced Nitrogen and Supplemented Amino Acids Nutrient Solution on the Nutritional Quality of Baby Green and Red Lettuce Grown in a Floating System
}

\author{
Pavlos Tsouvaltzis ${ }^{1, *}$, Dimitrios S. Kasampalis ${ }^{1}$, Danai-Christina Aktsoglou ${ }^{1}$, \\ Nikolaos Barbayiannis ${ }^{2}$ and Anastasios S. Siomos ${ }^{1}$ (D) \\ 1 Lab of Vegetable Crops, Department of Horticulture, Aristotle University, 54124 Thessaloniki, Greece; \\ kasampal@agro.auth.gr (D.S.K.); daktsogl@agro.auth.gr (D.-C.A.); siomos@agro.auth.gr (A.S.S.) \\ 2 Lab of Soil Science, Department of Hydraulics, Soil Science and Agricultural Engineering, \\ Aristotle University, 54124 Thessaloniki, Greece; nikbarba@agro.auth.gr \\ * Correspondence: ptsouv@agro.auth.gr; Tel.: +30-231-099-8802
}

Received: 27 May 2020; Accepted: 25 June 2020; Published: 27 June 2020

\begin{abstract}
Excessive nitrogen fertilization results in nitrate accumulation in leafy vegetables. Reducing the dose of mineral nitrogen or using alternate fertilizers lowers the nitrate accumulation; however, a critical minimum level of mineral nitrogen is necessary to maintain yield and nutritional quality. The aim of this study was to evaluate the effect of two levels of mineral nitrogen $(100 \%$ and $50 \%)$ and three levels of an amino acid solution $(0,0.3$, and $0.9 \%)$ in the nutrient solution of two baby lettuce cultivars (green and red) grown in a floating system. Nitrogen reduction did not affect yield (12.9-13.4 and 11.0-11.3 g/plant, respectively) but reduced nitrate accumulation (by 43 and 19\%, respectively) in both green and red lettuce, while enhancing phenolic content (by 28\%) and antioxidant capacity (by $69 \%$ ) in green lettuce and soluble solid (by 7\%) and total chlorophyll content (by $9 \%$ ) in red lettuce. Although nitrate accumulation was prevented $(<355 \mathrm{mg} / \mathrm{kg} \mathrm{FW})$ and most nutritional components increased in both lettuce types by amino acids supplementation, plant growth was negatively affected, especially in red lettuce, in both concentrations of amino acids (reduction by 9 and $35 \%$ in 0.3 and $0.9 \%$, respectively). In both lettuce types, proline content increased by $0.9 \%$ amino acids supplementation (by $45 \%$ ), implying a probable induction of a stress condition. Mineral nutrients were slightly affected by nitrogen reduction, which was probably perceived as an abiotic stress.
\end{abstract}

Keywords: soilless; nitrates; nitrogen use efficiency; protein hydrolysate; biostimulants; quality

\section{Introduction}

Modern agricultural management techniques in vegetable cultivation are target towards the intensive controlled production of superior nutritional quality products, along with the highest possible water and nitrogen use efficiency [1-3]. Soilless growth systems meet these requirements [4] and have been adapted and implemented by many growers [5]. Closed hydroponic systems lead to a great reduction of inputs, such as water and fertilizers and are suitable for the growth of leafy vegetables even without the use of substrates, as in the case of floating tray systems. This soilless system has many advantages, such as the low installation and operation costs, the use of high plant densities, and the absence of any substrate residues on the harvested products [6-8]. The lack of interactions among roots, soil, and soil-containing microorganisms $[9,10]$ renders this soilless system suitable to study in detail the actual needs of a plant in vivo in terms of water and nitrogen rates and forms requirements [11], along with the plant's response to biostimulant supplements, such as amino acids [12,13]. 
Nitrogen is the most essential mineral nutrient that promotes plant growth, yield, and a satisfactory income to growers. It is absorbed by the roots either as ammonium or nitrate ion, assimilated by the activity of nitrate reductase enzymes, and incorporated into amino acids that constitute the structural elements of proteins [11,14]. However, growers frequently use excessive rates of nitrogen fertilization in vegetable cultivation, fearing that the recommended doses may lead to deficiency [11,15], and ignoring the environmental pollution, production cost increase, and product quality deterioration issues $[1,3,11,16-19]$. Abundant nitrate availability leads to excessive absorption by the roots in larger quantities than those that nitrate reductase can convert into ammonium $[9,11,18]$, resulting in nitrate accumulation in the vacuoles of the cells [20]. This is a frequent occasion in leafy vegetables, which tend to accumulate excessive concentrations of nitrates in their tissues, which is regarded as a negative qualitative aspect from a nutritional standpoint because nitrate consumption by humans has been associated with various health hazards [4,11]. Therefore, as reviewed by Santamaria [4], the World Health Organization of the United Nations and the European Commission have set a maximum acceptable daily intake for $\mathrm{NO}_{3}$ of $3.7 \mathrm{mg} \mathrm{kg}^{-1}$ bodyweight, while the US Environmental Protection Agency has set this level higher at $7.0 \mathrm{mg} \mathrm{NO}_{3} \mathrm{~kg}^{-1}$ bodyweight per day.

Accumulation of nitrate in lettuce depends on growth season, due to light intensity variation during the year [9,21,22]; cultivar and type of lettuce [11,22-24]; nitrogen supply in terms of fertilizer form and doses applied [15,21,22,24-27]; and growth stage of the plants at harvest $[16,22,28]$. The use of supplemental lightning can be implemented only in greenhouses, and it is considered an expensive process [29], whereas recent selection strategies do not appear to have produced lower-nitrate-accumulating cultivars [28]. Manipulation of nitrogen fertilization in terms of rates of application and source type appears as the most applicable means to prevent nitrate accumulation in plants [18]. Replacement of part of the nitrate fertilizer with ammonium nitrogen is an advantageous practice to avoid nitrate accumulation without negative consequences on crop yield $[18,19,24,28,30]$. However, caution should be taken to ensure that a critical minimum level of mineral nitrogen for sustaining plant growth and crop yield is not surpassed and, additionally, mineral nitrogen manipulation does not induce nutritional quality deterioration $[1,2,8,26,31-36]$.

Recent research has proven that the use of organic fertilizers, such as green manure, cattle manure, wine distillery wastewater, or olive pomace compost, in lettuce crop production can be considered a valid and useful alternative source of organic nitrogen [3,15], but information regarding the application of amino acids, another source of organic nitrogen, is limited in the literature. Amino acids are easily absorbed by the roots in field [10,13,37-39] or soilless grown plants [40] and are transported via the xylem towards the upper plant organs. Uptake of multiple amino acids by plants is preferable as sources of reduced nitrogen at the expense of nitrate uptake [41], preventing nitrate accumulation in plant tissues [42]. However, this is in contradiction with the findings of other researchers, who claim that the amino acids do not have a significant contribution as sources of nitrogen, but they regulate thenitrate uptake and assimilation by affecting the enzyme activities that participate in plant nitrogen metabolism [40,43].

The addition of amino acids in the nutrient solution in soilless production of leafy vegetables has shown that as amino acid content [40] and mineral nutrient concentrations [18,44] in plant tissues increase, dry matter is either increased, decreased, or unaffected $[40,41]$ depending on the individual amino acids applied [44], but no information regarding other nutritional quality traits of the products has been examined. Detailed overviews on the definition and concept of protein hydrolysates, the main categories of plant biostimulants, as well as implications regarding the plant responses to them are provided by du Jardin [45] and Rouphael and Colla [46]. The benefits of amino acid supplements may help growers to adapt it as a practice in hydroponic production of vegetables in order to supply the market with baby leaf products of the highest nutritional value and the lowest potential health hazards, which are preferable to consumers. 
The aim of this study was to assess the possibility of reducing mineral nitrogen input and to evaluate the effect of a mixed amino acids solution supplement in the nutrient solution of two lettuce cultivars grown in floating trays to enhance product nutritional quality without reducing crop yield.

\section{Materials and Methods}

\subsection{Plant Material and Growth Conditions}

Leaf lettuce (Lactuca sativa L. var. crispa) seeds of a green (cv. Levistro) and a red cultivar (cv. Carmesi) were sown on expanded polystyrene trays at a plant density of 167 plants $\mathrm{m}^{-2}$, in a glass greenhouse during winter. Ten days after sowing, the trays with the germinated seeds were transferred to six tanks containing $50 \mathrm{~L}$ of nutrient solution each, which, apart from the nitrogen $(\mathrm{N})$, had the same mineral composition: P 62 mg/L, K 490 mg/L, Ca 190 mg/L, Mg 24 mg/L, S 202 mg/L, Fe 2236 g/L, Mn $275 \mu \mathrm{g} / \mathrm{L}, \mathrm{Zn} 262 \mu \mathrm{g} / \mathrm{L}, \mathrm{B} 324 \mu \mathrm{g} / \mathrm{L}, \mathrm{Cu} 48 \mu \mathrm{g} / \mathrm{L}$, and Mo $48 \mu \mathrm{g} / \mathrm{L}$. The above composition was prepared using potassium nitrate, calcium nitrate, phosphoric acid, magnesium sulfate (heptahydrate), iron EDDHA, manganese sulfate (monohydrate), zinc sulfate (heptahydrate), boric acid, copper sulfate (pentahydrate), and molybdenum trioxide chemicals.

In half of the tanks, $131 \mathrm{mg} / \mathrm{L} \mathrm{N}$ in nitrate form was added, corresponding to the N-sufficient nutrient solutions $(100 \% \mathrm{~N})$, while in the other half of the tanks, half the rate of $\mathrm{N}$ was added $(65.5 \mathrm{mg} / \mathrm{L})$, corresponding to the N-reduced nutrient solutions $(50 \% \mathrm{~N})$. A commercial protein hydrolysate solution product, Amino $16{ }^{\circledR}$ (EVYP LLP, Thessaloniki, Greece), which contains $11.3 \%$ L-amino acids, $4 \%$ total $\mathrm{N}$, and $25 \%$ organic matter, was diluted in two concentrations ( 0.3 and $0.9 \% \mathrm{v} / \mathrm{v})$ in two tanks with $100 \%$ $\mathrm{N}$ and in two tanks with 50\% N. According to the European Patent Office (Bulletin 2012/52, 2012) [47], the composition of the end-product of Amino $16^{\circledR}$ in terms of individual amino acids and their (\% $\mathrm{w} / \mathrm{w}$ ) concentration is alanine (0.59), arginine (0.75), aspartic acid (1.10), glutamic acid (1.33), glycine (0.32), histidine (0.31), isoleucine (0.75), leucine (2.60), lysine (0.39), methionine (0.25), phenylalanine (1.13), proline (0.95), serine (0.34), threonine (0.24), tyrosine (0.60), and valine (0.84). In the remaining two tanks, no Amino $16^{\circledR}$ was added.

During cultivation, the nutrient solutions were agitated frequently in order to avoid oxygen depletion, and the $\mathrm{pH}$ and electrical conductivity of the nutrient solutions were recorded. Before harvest, the length of roots that grew out of the bottom of the trays was measured with a ruler. Thirty plants per lettuce cultivar and per tank were harvested 37 days from the initiation of the experiment at the baby stage, and their individual weight was recorded. Afterwards, the plants of each cultivar were randomly divided in 3 groups with 10 plants per group and were homogenized in a waring blender for the assessment of nutritional quality.

\subsection{Nutritional Quality Assessment}

Dry matter was determined after drying about $40 \mathrm{~g}$ of blended material at $70{ }^{\circ} \mathrm{C}$ for $72 \mathrm{~h}$.

Total soluble solids were measured on a digital refractometer Atago PR-1 (Atago Co. Ltd., Tokyo, Japan).

For the extraction of nitrates, $2.5 \mathrm{~g}$ of the blended material were homogenized with $25 \mathrm{~mL}$ of deionized water. Nitrate content was determined in the filtrate following the method of Cataldo et al. [48].

For the determination of total soluble phenols and total antioxidant capacity, $5 \mathrm{~g}$ of the blended material were homogenized with $25 \mathrm{~mL} \mathrm{80 \%} \mathrm{methanol} \mathrm{and} \mathrm{centrifuged} \mathrm{at} \mathrm{5000 \times} \mathrm{g}$ for $20 \mathrm{~min}$. Total soluble phenols were determined following the method of Scalbert et al. [49], using gallic acid for the standard curve. Total antioxidant capacity was determined following the method of Brand-Williams et al. [50]. The radical scavenging capacity of DPPH, representing the total antioxidant capacity, was expressed as mg ascorbic acid equivalents per $\mathrm{g}$ fresh weight.

For the extraction of chlorophylls and carotenoids, $1 \mathrm{~g}$ of the blended material was mixed with $10 \mathrm{~mL} 80 \%$ acetone in plastic tubes with a cap and stored at $-20^{\circ} \mathrm{C}$. After thawing, the samples were 
vortexed, centrifuged at $14,000 \mathrm{rpm}$ for $10 \mathrm{~min}$ at $20^{\circ} \mathrm{C}$, and the supernatant was filtered in $25 \mathrm{~mL}$ volumetric vials. Another $10 \mathrm{~mL} 80 \%$ acetone were added to the residue, and the samples were shaken at $150 \mathrm{rpm}$ for $10 \mathrm{~min}$. The samples were filtered again and added to the previous filtrates. The vials were filled with $80 \%$ acetone, and the absorbance was measured in a spectrophotometer at 470,645 , and $663 \mathrm{~nm}$. The determination of $a, b$, total chlorophyll, and total carotenoids was performed according to Arnon [51].

Anthocyanin content was determined according to the method of Fuleki and Francis [52]. For calculation of the anthocyanin content ( $\mu \mathrm{g}$ per $\mathrm{g}$ fresh weight), the anthocyanin extinction coefficient of $984 \mathrm{~g}$ per $100 \mathrm{~mL}$ per $\mathrm{cm}$ was used.

For proline extraction, $0.1 \mathrm{~g}$ of frozen plant material chopped into small pieces was placed in $25 \mathrm{~mL}$ glass test tubes and in each tube $15 \mathrm{~mL}$ of $80 \%$ ethanol was added. The tubes with the plant material were incubated at $60{ }^{\circ} \mathrm{C}$ in a water bath for $30 \mathrm{~min}$. Free proline in the extract was measured with acid ninhydrin solution according to the method of Troll and Lindsley [53].

For the determination of mineral composition, the dry matter samples were ground and sieved through a 0.2-mm sieve. $\mathrm{N}$ was determined by the Kjeldahl method [54], P was determined by the molybdenum blue-ascorbic acid method [55], $\mathrm{K}$ and $\mathrm{Na}$ were determined by flame emission spectroscopy and were expressed as \% dry weight, and $\mathrm{Ca}, \mathrm{Mg}, \mathrm{Mn}, \mathrm{Fe}, \mathrm{Cu}$, and $\mathrm{Zn}$ were measured by atomic absorption spectroscopy and expressed as $\mu \mathrm{g} / \mathrm{g}$ dry weight.

\subsection{Statistical Analysis}

A completely randomized design was used in the greenhouse study, comprising 30 replications per treatment with one plant per replication, while a completely randomized design with 3 replications (10 lettuce plants per replication) was used for the determination of the nutritional quality and mineral composition. Analysis of variance (ANOVA) was performed in MSTAT (Michigan State University) and mean separation was done with Duncan multiple range test $(p<0.05)$. The effect of each factor was calculated as the percent of total variance $(\% T V)=$ Means Square factor/Mean Square total.

\section{Results}

The $\mathrm{pH}$ of the 50 and $100 \% \mathrm{~N}$ nutrient solutions, free of any amino acid supplement, was 6.50 during the whole production period, without significant fluctuations (Figure 1A). On the other hand, the addition of 0.3 or $0.9 \%$ amino acids in the nutrient solution resulted in a decrease of the $\mathrm{pH}$ to around 4.0 and 3.4, respectively, which was followed by a sigmoidal pattern of increase, reaching the control levels after 10 and 24 days, respectively, regardless of the $\mathrm{N}$ concentration; the $\mathrm{pH}$ even increased beyond that of the control while reaching the end of the production cycle. The electrical conductivity of the nutrient solutions without receiving any Amino16 ${ }^{\circledR}$ ewas 3.4, while the addition of 0.3 and $0.9 \%$ amino acids solutions shifted the electrical conductivity (EC) up to 4.4 and $6.4 \mathrm{mS} / \mathrm{cm}$, respectively, (Figure 1B). These initial levels remained constant for the whole growing period.

In order to elucidate the effect of $\mathrm{N}$ and amino acids concentrations in the nutrient solution, data were processed within each lettuce type (green and red).

Reducing N by $50 \%$ in the nutrient solution of the green lettuce had no effect on plant weight, but it resulted in increased ( 2.5 times) root length compared with that of the control plants $(100 \% \mathrm{~N})$ (Table 1). Furthermore, plants grown on $50 \% \mathrm{~N}$ accumulated almost half the nitrate content and higher phenol content and antioxidant capacity in comparison to that of the control plants (Table 2). Moreover, the reduction of $\mathrm{N}$ in the nutrient solution resulted in reduced total $\mathrm{N}, \mathrm{Fe}$, and $\mathrm{Mn}$ absorption by the roots (Table 3). As an average of the two $\mathrm{N}$ levels in the nutrient solution (50 or 100\%), the intermediate concentration $(0.3 \%)$ of amino acids solution supplement did not affect either the plant weight nor root elongation (Table 1) of plants, but reduced nitrate and increased chlorophyll (Table 2) and Zn (Table 3) content. When an increased concentration ( $0.9 \%)$ of amino acids solution supplement was used, the plant weight decreased, and root growth was entirely inhibited (Table 1). Similar to the $0.3 \%$ supplement, the addition of the higher $(0.9 \%)$ amino acid concentration resulted again in decreased 
nitrate accumulation and increased content of total chlorophylls and $\mathrm{Zn}$ and, furthermore, in increased carotenoid, proline, P, Na, Fe, and Zn content (Tables 2 and 3).

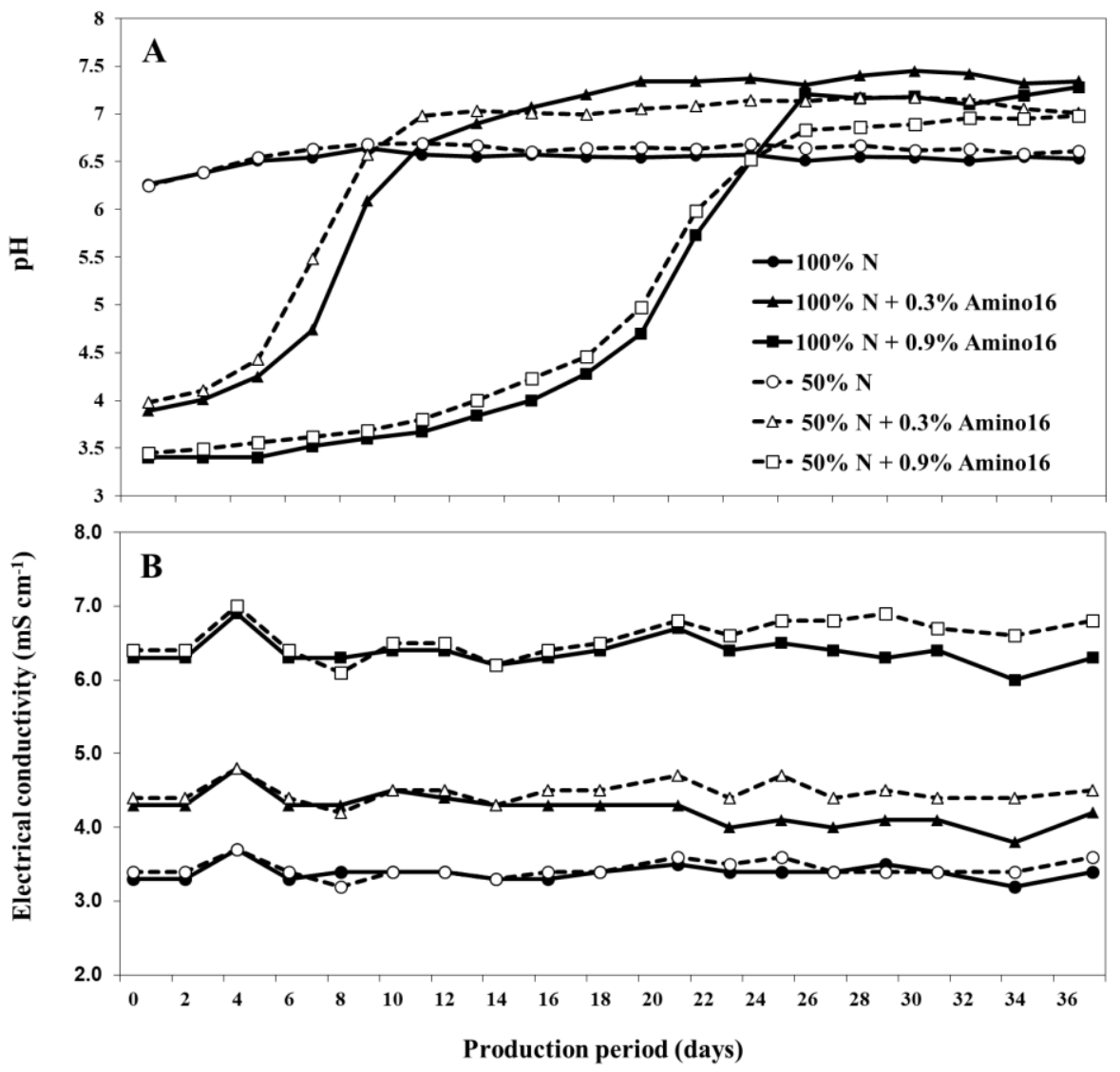

Figure 1. The $\mathrm{pH}(\mathbf{A})$ and the electrical conductivity (B) of the nutrient solutions supplied with 131 $(100 \%)$ or $65.5 \mathrm{mg} / \mathrm{L}(50 \%) \mathrm{N}$ and supplemented with $0,0.3$, or $0.9 \%$ Amino $16{ }^{\circledR}$ during growth of baby green and red loose-leaf lettuce.

Table 1. Analysis of variance and mean comparison of plant weight and root length of green baby lettuce grown in $131(100 \%)$ or $65.5 \mathrm{mg} / \mathrm{L}(50 \%) \mathrm{N}$ supplemented with $0,0.3$, or $0.9 \%$ Amino16.

\begin{tabular}{|c|c|c|c|c|c|}
\hline \multirow[t]{2}{*}{ Nitrogen $(\%)$} & \multirow{2}{*}{$\begin{array}{c}\text { Amino16 (\%) } \\
\text { Source of variation }\end{array}$} & \multicolumn{2}{|c|}{ Plant Weight (g) } & \multicolumn{2}{|c|}{ Root Length $(\mathrm{cm})$} \\
\hline & & $p$ & $\% \mathrm{TV}^{\mathrm{s}}$ & $p$ & $\% \mathrm{TV}$ \\
\hline & $(\mathrm{N})$ Nitrogen $\mathrm{v}$ & & & $* * *$ & 34 \\
\hline & (A) Amino $16^{\mathrm{u}}$ & $* * * \mathrm{r}$ & 85 & $* * *$ & 45 \\
\hline & $\mathrm{N} \times \mathrm{A}$ & & & $* * *$ & 20 \\
\hline 100 & & $13.38^{z}$ & & $1.51 \mathrm{~b}$ & \\
\hline \multirow[t]{4}{*}{50} & & 12.85 & & $3.70 \mathrm{a}$ & \\
\hline & 0 & $14.61 \mathrm{a}^{\mathrm{y}}$ & & $3.68 \mathrm{a}$ & \\
\hline & 0.3 & $13.80 \mathrm{a}$ & & $4.15 \mathrm{a}$ & \\
\hline & 0.9 & $10.95 \mathrm{~b}$ & & $0.00 \mathrm{~b}$ & \\
\hline \multirow[t]{3}{*}{100} & 0 & 14.55 & & $3.14 \mathrm{c}$ & \\
\hline & 0.3 & 14.41 & & $1.41 \mathrm{~d}$ & \\
\hline & 0.9 & 11.19 & & $0.00 \mathrm{e}$ & \\
\hline \multirow[t]{3}{*}{50} & 0 & 14.67 & & $4.22 \mathrm{~b}$ & \\
\hline & 0.3 & 13.19 & & $6.89 \mathrm{a}$ & \\
\hline & 0.9 & 10.70 & & $0.00 \mathrm{e}$ & \\
\hline
\end{tabular}

z: each value is the mean of 30 replications; y: numbers in the same column followed by different letter are significantly different, according to the LSD $(p<0.05)$; v: 50 or $100 \%$ nitrogen in the nutrient solution; u: $0,0.3$ or $0.9 \%$ Amino16 in the nutrient solution; s: total variance; $\mathrm{r}:{ }^{*}, * *{ }^{* * *}$ significant effect at $p<0.05,0.01$ or 0.001 , respectively. 
Table 2. Analysis of variance and mean comparison of dry matter, nitrate, soluble solid (SSC), phenolic, total chlorophyll, total carotenoid, and proline content, as well as total antioxidant capacity of green baby lettuce grown in $131(100 \%)$ or $65.5 \mathrm{mg} / \mathrm{L} \mathrm{(50 \% )} \mathrm{N} \mathrm{supplemented} \mathrm{with} 0,0.3$, or $0.9 \%$ Amino $16^{\circledR}$.

\begin{tabular}{|c|c|c|c|c|c|c|c|c|c|c|c|c|c|c|c|c|c|}
\hline \multirow[t]{2}{*}{$\begin{array}{l}\text { Nitrogen } \\
(\%)\end{array}$} & \multirow{2}{*}{$\begin{array}{c}\text { Amino16 (\%) } \\
\begin{array}{c}\text { Source of } \\
\text { variation }\end{array} \\
\end{array}$} & \multicolumn{2}{|c|}{ Dry Matter (\%) } & \multicolumn{2}{|c|}{$\begin{array}{c}\text { Nitrates }(\mathrm{mg} / \mathrm{kg} \\
\text { FW) }\end{array}$} & \multicolumn{2}{|c|}{ SSC (\%) } & \multicolumn{2}{|c|}{$\begin{array}{l}\text { Phenols ( } \mu \text { g } \\
\text { GAE/g FW) }\end{array}$} & \multicolumn{2}{|c|}{$\begin{array}{c}\text { Antioxidant } \\
\text { Capacity (mg } \\
\text { AEAC/100 g FW) }\end{array}$} & \multicolumn{2}{|c|}{$\begin{array}{l}\text { Chlorophyll } \\
\text { ( } \mu \mathrm{g} / \mathrm{g} \text { FW) }\end{array}$} & \multicolumn{2}{|c|}{$\begin{array}{l}\text { Carotenoids } \\
(\mu \mathrm{g} / \mathrm{g} \text { FW) }\end{array}$} & \multicolumn{2}{|c|}{ Prolines (mM) } \\
\hline & & $p$ & $\% \mathrm{TV}^{\mathrm{s}}$ & $p$ & $\% \mathrm{TV}$ & $p$ & $\% \mathrm{TV}$ & $p$ & $\% \mathrm{TV}$ & $p$ & $\% \mathrm{TV}$ & $p$ & $\% \mathrm{TV}$ & $p$ & $\% \mathrm{TV}$ & $p$ & $\% \mathrm{TV}$ \\
\hline & Nitrogen $(\mathrm{N})^{\mathrm{v}}$ & & & $* * * \mathrm{r}$ & 47 & & & $* * *$ & 71 & $* * *$ & 82 & & & & & & \\
\hline & Amino16 (A16) u & & & $* * *$ & 35 & & & & & & & $* * *$ & 80 & * & 64 & $* * *$ & 88 \\
\hline & $\mathrm{N} \times \mathrm{A} 16$ & & & $* * *$ & 17 & $* * *$ & 58 & $*$ & 20 & & & & & & & & \\
\hline 100 & & $4.63^{z}$ & & $469 \mathrm{a}^{\mathrm{y}}$ & & 3.02 & & $0.050 \mathrm{~b}$ & & $2.91 \mathrm{~b}$ & & 304 & & 67.7 & & 0.00370 & \\
\hline \multirow[t]{4}{*}{50} & & 4.75 & & $269 \mathrm{~b}$ & & 3.14 & & $0.064 \mathrm{a}$ & & $4.93 \mathrm{a}$ & & 292 & & 65.7 & & 0.00394 & \\
\hline & 0 & 4.75 & & $519 \mathrm{a}$ & & 3.06 & & 0.058 & & 4.13 & & $258 \mathrm{~b}$ & & $62.1 \mathrm{~b}$ & & $0.00280 \mathrm{~b}$ & \\
\hline & 0.3 & 4.60 & & $233 c$ & & 2.97 & & 0.054 & & 3.83 & & $308 \mathrm{a}$ & & $67.1 \mathrm{~b}$ & & $0.00335 \mathrm{~b}$ & \\
\hline & 0.9 & 4.71 & & $355 \mathrm{~b}$ & & 3.22 & & 0.060 & & 3.80 & & $328 \mathrm{a}$ & & $70.8 \mathrm{a}$ & & $0.00531 \mathrm{a}$ & \\
\hline \multirow[t]{3}{*}{100} & 0 & 4.69 & & $729 a$ & & $2.78 \mathrm{~b}$ & & $0.044 \mathrm{c}$ & & 2.49 & & 256 & & 61.0 & & 0.00298 & \\
\hline & 0.3 & 4.72 & & $320 \mathrm{~b}$ & & $3.10 \mathrm{ab}$ & & $0.052 \mathrm{bc}$ & & 3.42 & & 314 & & 68.6 & & 0.00321 & \\
\hline & 0.9 & 4.47 & & $358 \mathrm{~b}$ & & $3.17 \mathrm{ab}$ & & $0.053 \mathrm{bc}$ & & 2.84 & & 343 & & 73.3 & & 0.00491 & \\
\hline \multirow[t]{3}{*}{50} & 0 & 4.81 & & $308 \mathrm{~b}$ & & $3.33 \mathrm{a}$ & & $0.072 \mathrm{a}$ & & 5.78 & & 261 & & 63.2 & & 0.00262 & \\
\hline & 0.3 & 4.48 & & $147 \mathrm{c}$ & & $2.83 \mathrm{~b}$ & & $0.055 \mathrm{bc}$ & & 4.25 & & 303 & & 65.6 & & 0.00349 & \\
\hline & 0.9 & 4.96 & & $352 \mathrm{~b}$ & & $3.27 \mathrm{a}$ & & $0.066 \mathrm{ab}$ & & 4.77 & & 313 & & 68.3 & & 0.00570 & \\
\hline
\end{tabular}

z: Each value is the mean of 3 replications; y: numbers in the same column follwed by different letter are sugnificantly different, according to the LSD ( $p<0.05)$; v: 50 or $100 \%$ nitrogen in the nutrient solution; $\mathrm{u}: 0,0.3$ or $0.9 \%$ Amino16 in the nutrient solution; s: total variance; r: ${ }^{* * *}{ }^{* * *}$ significant effect at $p<0.05,0.01$ or 0.001 , respectively. 
Table 3. Analysis of variance and mean comparison of mineral (N, K, P, Na, Ca, Mg, Fe, Mn, Cu, and Zn) content of green baby lettuce grown in 131 (100\%) or $65.5 \mathrm{mg} / \mathrm{L}(50 \%) \mathrm{N}$ supplemented with $0,0.3$, or $0.9 \%$ Amino $16^{\circledR}$.

\begin{tabular}{|c|c|c|c|c|c|c|c|c|c|c|c|c|c|c|c|c|c|c|c|c|c|}
\hline \multirow[t]{2}{*}{$\begin{array}{c}\text { Nitrogen } \\
(\%)\end{array}$} & \multirow{2}{*}{$\begin{array}{c}\text { Amino16 (\%) } \\
\begin{array}{c}\text { Source of } \\
\text { variation }\end{array}\end{array}$} & \multicolumn{2}{|c|}{ N (\% DW) } & \multicolumn{2}{|c|}{ K (\% DW) } & \multicolumn{2}{|c|}{$P(\% \mathrm{DW})$} & \multicolumn{2}{|c|}{$\mathrm{Na}(\% \mathrm{DW})$} & \multicolumn{2}{|c|}{$\begin{array}{c}\text { Ca }(\mu \mathrm{g} / \mathrm{g} \\
\text { DW) }\end{array}$} & \multicolumn{2}{|c|}{$\begin{array}{c}\mathrm{Mg}(\mu \mathrm{g} / \mathrm{g} \\
\mathrm{DW})\end{array}$} & \multicolumn{2}{|c|}{ Fe ( $\mu g / g$ DW) } & \multicolumn{2}{|c|}{$\begin{array}{c}\mathrm{Mn}(\mu \mathrm{g} / \mathrm{g} \\
\mathrm{DW})\end{array}$} & \multicolumn{2}{|c|}{$\mathrm{Cu}(\mu \mathrm{g} / \mathrm{g}$ DW) } & \multicolumn{2}{|c|}{$\mathrm{Zn}(\mu \mathrm{g} / \mathrm{g}$ DW $)$} \\
\hline & & $p$ & $\begin{array}{c}\% \\
\mathrm{TV}^{\mathrm{s}}\end{array}$ & $p$ & $\begin{array}{c}\% \\
\text { TV }\end{array}$ & $p$ & $\begin{array}{l}\% \\
\text { TV }\end{array}$ & $p$ & $\begin{array}{c}\% \\
\text { TV }\end{array}$ & $p$ & $\begin{array}{c}\% \\
\text { TV }\end{array}$ & $p$ & $\begin{array}{l}\% \\
\text { TV }\end{array}$ & $p$ & $\begin{array}{l}\% \\
\text { TV }\end{array}$ & $p$ & $\begin{array}{c}\% \\
\text { TV }\end{array}$ & $p$ & $\begin{array}{l}\% \\
\text { TV }\end{array}$ & $p$ & $\begin{array}{c}\% \\
\text { TV }\end{array}$ \\
\hline & Nitrogen $(\mathrm{N})^{\mathrm{v}}$ & $* * \mathrm{r}$ & 68 & & & & & & & & & & & $* *$ & 39 & $* * *$ & 13 & & & & \\
\hline & Amino16 (A16) ${ }^{u}$ & & & & & ** & 61 & $* * *$ & 95 & & & & & $* * *$ & 51 & $* * *$ & 78 & & & $* * *$ & 47 \\
\hline & $\mathrm{N} \times \mathrm{A} 16$ & & & & & & & & & & & & & & & $* * *$ & 8 & $* * *$ & 75 & $* * *$ & 41 \\
\hline 100 & & $4.61^{\mathrm{z}} \mathrm{a}^{\mathrm{y}}$ & & 7.15 & & 0.643 & & 0.763 & & 0.867 & & 0.428 & & $32.73 \mathrm{a}$ & & $4.76 \mathrm{a}$ & & 3.14 & & 7.51 & \\
\hline \multirow{4}{*}{50} & & $4.20 \mathrm{~b}$ & & 6.94 & & 0.621 & & 0.791 & & 0.830 & & 0.322 & & $26.36 \mathrm{~b}$ & & $4.24 \mathrm{~b}$ & & 2.72 & & 7.78 & \\
\hline & 0 & 4.20 & & 7.20 & & $0.594 \mathrm{~b}$ & & $0.313 \mathrm{~b}$ & & 0.888 & & 0.313 & & $24.41 \mathrm{~b}$ & & $3.71 \mathrm{~b}$ & & 2.89 & & $7.04 \mathrm{~b}$ & \\
\hline & 0.3 & 4.47 & & 6.83 & & $0.617 \mathrm{~b}$ & & $0.483 \mathrm{~b}$ & & 0.769 & & 0.343 & & $27.28 \mathrm{~b}$ & & $3.95 \mathrm{~b}$ & & 3.26 & & $7.93 \mathrm{a}$ & \\
\hline & 0.9 & 4.54 & & 7.10 & & $0.685 a$ & & $1.536 \mathrm{a}$ & & 0.888 & & 0.469 & & $36.95 \mathrm{a}$ & & $5.84 \mathrm{a}$ & & 2.64 & & $7.96 \mathrm{a}$ & \\
\hline \multirow[t]{3}{*}{100} & 0 & 4.55 & & 7.36 & & 0.603 & & 0.329 & & 0.857 & & 0.327 & & 27.24 & & $3.78 \mathrm{c}$ & & $3.22 \mathrm{ab}$ & & $7.39 \mathrm{bcd}$ & \\
\hline & 0.3 & 4.57 & & 6.64 & & 0.604 & & 0.339 & & 0.758 & & 0.384 & & 28.49 & & $3.96 \mathrm{c}$ & & $2.39 \mathrm{bc}$ & & $7.33 \mathrm{~cd}$ & \\
\hline & 0.9 & 4.71 & & 7.43 & & 0.723 & & 1.622 & & 0.985 & & 0.573 & & 42.47 & & $6.55 \mathrm{a}$ & & $3.81 \mathrm{ab}$ & & $7.81 \mathrm{abc}$ & \\
\hline \multirow[t]{3}{*}{50} & 0 & 3.85 & & 7.03 & & 0.586 & & 0.297 & & 0.919 & & 0.299 & & 21.58 & & $3.63 c$ & & $2.55 \mathrm{abc}$ & & $6.69 \mathrm{~d}$ & \\
\hline & 0.3 & 4.37 & & 7.01 & & 0.630 & & 0.627 & & 0.779 & & 0.302 & & 26.07 & & $3.94 \mathrm{c}$ & & $4.12 \mathrm{a}$ & & $8.52 \mathrm{a}$ & \\
\hline & 0.9 & 4.37 & & 6.77 & & 0.648 & & 1.449 & & 0.791 & & 0.366 & & 31.42 & & $5.14 \mathrm{~b}$ & & $1.48 \mathrm{c}$ & & $8.11 \mathrm{ab}$ & \\
\hline
\end{tabular}

z: Each value is the mean of 3 replications; y: numbers in the same column follwed by different letter are sugnificantly different, according to the LSD ( $p<0.05)$; v: 50 or $100 \%$ nitrogen in the nutrient solution; $\mathrm{u}: 0,0.3$ or $0.9 \%$ Amino16 in the nutrient solution; s: total variance; $\mathrm{r}:{ }^{*}, * *, * * *$ significant effect at $p<0.05,0.01$ or 0.001 , respectively. 
In the red lettuce, the reduction of $\mathrm{N}$ by $50 \%$ in the nutrient solution did not affect the baby plants weight, although it significantly increased the root length compared with that of the control plants $(100 \% \mathrm{~N})$ (Table 4), similar to the results for the green type plants. Furthermore, the reduction of $\mathrm{N}$ by $50 \%$ resulted in higher dry matter, soluble solids, and total chlorophyll content, and lower nitrate, $\mathrm{K}$, $\mathrm{Na}, \mathrm{Mg}$, and Fe content of plants (Tables 5 and 6). As an average of the two $\mathrm{N}$ levels in the nutrient solution (50 or $100 \%$ ), the addition of amino acids reduced both plant weight and root growth in a proportional rate to the concentration (Table 4). Both amino acid doses reduced nitrate, $\mathrm{K}$ and $\mathrm{Ca}$ content, and increased phenolic, chlorophyll, carotenoid, and $\mathrm{Na}$ content, as well as total antioxidant capacity (Tables 5 and 6$)$. The higher amino acid concentration $(0.9 \%)$ also resulted in increased dry matter, soluble solid, proline, and Fe content (Tables 5 and 6).

Table 4. Analysis of variance and mean comparison of plant weight and root length of red baby lettuce grown in $131(100 \%)$ or $65.5 \mathrm{mg} / \mathrm{L}(50 \%) \mathrm{N}$ supplemented with $0,0.3$, or $0.9 \%$ Amino16 ${ }^{\circledR}$.

\begin{tabular}{|c|c|c|c|c|c|}
\hline \multirow[t]{2}{*}{ Nitrogen (\%) } & \multirow{2}{*}{$\begin{array}{c}\text { Amino16 (\%) } \\
\text { Source of variation }\end{array}$} & \multicolumn{2}{|c|}{ Plant Weight (g) } & \multicolumn{2}{|c|}{ Root Length (cm) } \\
\hline & & $p$ & $\% \mathrm{TV}^{\mathrm{s}}$ & $p$ & $\% \mathrm{TV}$ \\
\hline & $(\mathrm{N})$ Nitrogen $\mathrm{v}$ & & & $* * *$ & 9 \\
\hline & (A) Amino $16^{\mathrm{u}}$ & $* * * \mathrm{r}$ & 93 & $* * *$ & 79 \\
\hline & $\mathrm{N} \times \mathrm{A}$ & & & $* * *$ & 11 \\
\hline 100 & & $10.99^{z}$ & & $3.80 \mathrm{~b}$ & \\
\hline \multirow[t]{4}{*}{50} & & 11.30 & & $5.51 \mathrm{a}$ & \\
\hline & 0 & $13.03 \mathrm{~b}^{\mathrm{y}}$ & & $8.78 \mathrm{a}$ & \\
\hline & 0.3 & $11.89 \mathrm{~b}$ & & $5.19 \mathrm{~b}$ & \\
\hline & 0.9 & $8.51 \mathrm{c}$ & & $0.00 \mathrm{c}$ & \\
\hline \multirow[t]{3}{*}{100} & 0 & 12.97 & & $8.86 \mathrm{a}$ & \\
\hline & 0.3 & 11.25 & & $2.55 \mathrm{~b}$ & \\
\hline & 0.9 & 8.76 & & $0.00 \mathrm{c}$ & \\
\hline \multirow[t]{3}{*}{50} & 0 & 13.10 & & $8.70 \mathrm{a}$ & \\
\hline & 0.3 & 12.53 & & $7.83 \mathrm{a}$ & \\
\hline & 0.9 & 8.27 & & $0.00 \mathrm{c}$ & \\
\hline
\end{tabular}

$\mathrm{z}$ : each value is the mean of 30 replications; y: numbers in the same column followed by different letter are significantly different, according to the LSD ( $p<0.05) ; \mathrm{v}$ : 50 or $100 \%$ nitrogen in the nutrient solution; u: $0,0.3$ or $0.9 \%$ Amino16 in the nutrient solution; s: total variance; $\mathrm{r}:{ }^{*},{ }^{* *},{ }^{* * *}$ significant effect at $p<0.05,0.01$ or 0.001 , respectively. 
Table 5. Analysis of variance and mean comparison of dry matter, nitrate, soluble solid (SSC), phenolic, total chlorophyll, total carotenoid, anthocyanin, and proline content, as well as total antioxidant capacity of red baby lettuce grown in 131 (100\%) or $65.5 \mathrm{mg} / \mathrm{L} \mathrm{(50 \% )} \mathrm{N} \mathrm{supplemented} \mathrm{with} 0,0.3$, or $0.9 \%$ Amino $16{ }^{\circledR}$.

\begin{tabular}{|c|c|c|c|c|c|c|c|c|c|c|c|c|c|c|c|c|c|c|c|}
\hline \multirow[t]{2}{*}{$\begin{array}{l}\text { Nitrogen } \\
(\%)\end{array}$} & \multirow{2}{*}{$\begin{array}{c}\text { Amino16 (\%) } \\
\text { Source of variation }\end{array}$} & \multicolumn{2}{|c|}{ Dry Matter (\%) } & \multicolumn{2}{|c|}{$\begin{array}{c}\text { Nitrates } \\
(\mathrm{mg} / \mathrm{kg} \text { FW) }\end{array}$} & \multicolumn{2}{|c|}{ SSC (\%) } & \multicolumn{2}{|c|}{$\begin{array}{l}\text { Phenols ( } \mu g \\
\text { GAE/g FW) }\end{array}$} & \multicolumn{2}{|c|}{$\begin{array}{c}\text { Antioxidant } \\
\text { Capacity (mg } \\
\text { AEAC/100 g FW) }\end{array}$} & \multicolumn{2}{|c|}{$\begin{array}{c}\text { Chlorophyll } \\
\text { ( } \mu \mathrm{g} / \mathrm{g} \text { FW) }\end{array}$} & \multicolumn{2}{|c|}{$\begin{array}{l}\text { Carotenoids } \\
(\mu \mathrm{g} / \mathrm{g} \text { FW) }\end{array}$} & \multicolumn{2}{|c|}{$\begin{array}{l}\text { Anthocyanins } \\
(\mu \mathrm{g} / \mathrm{g} \text { FW) }\end{array}$} & \multicolumn{2}{|c|}{ Prolines (mM) } \\
\hline & & $p$ & $\begin{array}{c}\% \\
\% \\
\mathrm{TV}^{\mathrm{s}}\end{array}$ & $p$ & $\begin{array}{c}\% \\
\text { TV }\end{array}$ & $p$ & $\begin{array}{c}\% \\
\text { TV }\end{array}$ & $p$ & $\begin{array}{c}\% \\
\text { TV }\end{array}$ & $p$ & $\% \mathrm{TV}$ & $p$ & $\begin{array}{c}\% \\
\text { TV }\end{array}$ & $p$ & $\begin{array}{c}\% \\
\text { TV }\end{array}$ & $p$ & $\begin{array}{c}\% \\
\text { TV }\end{array}$ & $p$ & $\begin{array}{l}\% \\
\text { TV }\end{array}$ \\
\hline & Nitrogen $(\mathrm{N})^{\mathrm{v}}$ & $* * \mathrm{r}$ & 28 & $* * *$ & 20 & ** & 30 & & & & & $*$ & 8 & & & & & & \\
\hline & Amino16 (A16) ${ }^{\mathrm{u}}$ & $* * *$ & 45 & $* * *$ & 80 & $* * *$ & 57 & $* * *$ & 77 & $* * *$ & 76 & $* * *$ & 86 & $* * *$ & 81 & * & 69 & ** & 82 \\
\hline & $\mathrm{N} \times \mathrm{A} 16$ & $* * *$ & 25 & & & & & * & 17 & $*$ & 19 & & & & & & & & \\
\hline 100 & & $4.38^{\mathrm{z}} \mathrm{b}^{\mathrm{y}}$ & & $398 \mathrm{a}$ & & $2.72 \mathrm{~b}$ & & 0.157 & & 10.69 & & $453 \mathrm{~b}$ & & 81.83 & & 0.026 & & 0.00326 & \\
\hline \multirow[t]{4}{*}{50} & & $5.15 \mathrm{a}$ & & $321 \mathrm{~b}$ & & $2.90 \mathrm{a}$ & & 0.163 & & 11.18 & & $496 \mathrm{a}$ & & 83.99 & & 0.030 & & 0.00317 & \\
\hline & 0 & $4.16 \mathrm{~b}$ & & $499 \mathrm{a}$ & & $2.67 \mathrm{~b}$ & & $0.105 c$ & & $6.01 \mathrm{~b}$ & & $355 c$ & & $76.86 \mathrm{~b}$ & & $0.036 \mathrm{a}$ & & $0.00290 \mathrm{~b}$ & \\
\hline & 0.3 & $4.36 \mathrm{~b}$ & & $250 \mathrm{~b}$ & & $2.70 \mathrm{~b}$ & & $0.168 \mathrm{~b}$ & & 11.96 a & & $466 \mathrm{~b}$ & & 83.28 a & & $0.027 \mathrm{ab}$ & & $0.00308 \mathrm{~b}$ & \\
\hline & 0.9 & $5.77 \mathrm{a}$ & & $330 c$ & & $3.07 \mathrm{a}$ & & $0.206 \mathrm{a}$ & & $14.83 \mathrm{a}$ & & $604 \mathrm{a}$ & & $88.59 a$ & & $0.020 \mathrm{~b}$ & & $0.00367 \mathrm{a}$ & \\
\hline \multirow[t]{3}{*}{100} & 0 & $4.20 \mathrm{~b}$ & & 545 & & 2.53 & & $0.095 \mathrm{c}$ & & $5.23 c$ & & 348 & & 75.74 & & 0.035 & & 0.00294 & \\
\hline & 0.3 & $4.30 \mathrm{~b}$ & & 291 & & 2.70 & & $0.191 \mathrm{ab}$ & & $14.16 \mathrm{ab}$ & & 460 & & 81.36 & & 0.024 & & 0.00319 & \\
\hline & 0.9 & $4.64 \mathrm{~b}$ & & 358 & & 2.93 & & $0.184 \mathrm{ab}$ & & $12.66 \mathrm{ab}$ & & 551 & & 88.39 & & 0.017 & & 0.00365 & \\
\hline \multirow[t]{3}{*}{50} & 0 & $4.13 \mathrm{~b}$ & & 453 & & 2.80 & & $0.116 \mathrm{c}$ & & $6.79 \mathrm{c}$ & & 361 & & 77.98 & & 0.036 & & 0.00286 & \\
\hline & 0.3 & $4.41 \mathrm{~b}$ & & 208 & & 2.70 & & $0.144 \mathrm{bc}$ & & $9.76 \mathrm{bc}$ & & 471 & & 85.20 & & 0.029 & & 0.00298 & \\
\hline & 0.9 & $6.90 \mathrm{a}$ & & 302 & & 3.20 & & $0.228 \mathrm{a}$ & & $16.99 \mathrm{a}$ & & 657 & & 88.78 & & 0.024 & & 0.00369 & \\
\hline
\end{tabular}

z: Each value is the mean of 3 replications; y: numbers in the same column follwed by different letter are sugnificantly different, according to the LSD ( $p<0.05)$; v: 50 or $100 \%$ nitrogen in the nutrient solution; u: $0,0.3$ or $0.9 \%$ Amino16 in the nutrient solution; s: total variance; r: ${ }^{*}{ }^{* *}, * * *$ significant effect at $p<0.05,0.01$ or 0.001 , respectively. 
Table 6. Analysis of variance and mean comparison of mineral (N, K, P, Na, Ca, Mg, Fe, Mn, Cu, and Zn) content of red baby lettuce grown in 131 (100\%) or $65.5 \mathrm{mg} / \mathrm{L}$ (50\%) N supplemented with $0,0.3$, or $0.9 \%$ Amino $16^{\circledR}$.

\begin{tabular}{|c|c|c|c|c|c|c|c|c|c|c|c|c|c|c|c|c|c|c|c|c|c|}
\hline \multirow[t]{2}{*}{$\begin{array}{l}\text { Nitrogen } \\
(\%)\end{array}$} & \multirow{2}{*}{\begin{tabular}{|c|} 
Amino16 (\%) \\
$\begin{array}{l}\text { Source of } \\
\text { variation }\end{array}$ \\
\end{tabular}} & \multicolumn{2}{|c|}{ N (\% DW) } & \multicolumn{2}{|c|}{ K (\% DW) } & \multicolumn{2}{|c|}{ P (\% DW) } & \multicolumn{2}{|c|}{$\mathrm{Na}(\% \mathrm{DW})$} & \multicolumn{2}{|c|}{$\begin{array}{c}\mathrm{Ca}(\mu \mathrm{g} / \mathrm{g} \\
\text { DW) }\end{array}$} & \multicolumn{2}{|c|}{$\begin{array}{c}M g(\mu \mathrm{g} / \mathrm{g} \\
\text { DW) }\end{array}$} & \multicolumn{2}{|c|}{$\begin{array}{c}\mathrm{Fe}(\mu \mathrm{g} / \mathrm{g} \\
\text { DW) }\end{array}$} & \multicolumn{2}{|c|}{$\begin{array}{c}\text { Mn }(\mu \mathrm{g} / \mathrm{g} \\
\text { DW) }\end{array}$} & \multicolumn{2}{|c|}{$\begin{array}{c}\mathrm{Cu}(\mu \mathrm{g} / \mathrm{g} \\
\mathrm{DW})\end{array}$} & \multicolumn{2}{|c|}{$\begin{array}{c}\mathrm{Zn}(\mu \mathrm{g} / \mathrm{g} \\
\mathrm{DW})\end{array}$} \\
\hline & & $p$ & $\begin{array}{c}\% \\
\text { TV }^{\mathrm{s}}\end{array}$ & $p$ & $\begin{array}{c}\% \\
\text { TV }\end{array}$ & $p$ & $\begin{array}{c}\% \\
\text { TV }\end{array}$ & $p$ & $\begin{array}{c}\% \\
\text { TV }\end{array}$ & $p$ & $\begin{array}{c}\% \\
\text { TV }\end{array}$ & $p$ & $\begin{array}{c}\% \\
\text { TV }\end{array}$ & $p$ & $\begin{array}{c}\% \\
\text { TV }\end{array}$ & $p$ & $\begin{array}{l}\% \\
\text { TV }\end{array}$ & $p$ & $\begin{array}{c}\% \\
\text { TV }\end{array}$ & $p$ & $\begin{array}{l}\% \\
\text { TV }\end{array}$ \\
\hline & Nitrogen $(\mathrm{N})^{\mathrm{v}}$ & & & $* \mathrm{r}$ & 25 & & & $* * *$ & 5 & & & $* *$ & 84 & $* * *$ & 42 & & & & & & \\
\hline & Amino16 (A16) ${ }^{\mathrm{u}}$ & & & $* * *$ & 66 & & & $* * *$ & 95 & $* *$ & 64 & & & $* * *$ & 52 & $* * *$ & 76 & & & & \\
\hline & $\mathrm{N} \times \mathrm{A} 16$ & & & & & & & $*$ & 1 & & & & & & & $*$ & 18 & & & * & 60 \\
\hline 100 & & $5.37^{z}$ & & $9.00 \mathrm{a}^{\mathrm{y}}$ & & 0.637 & & $0.657 \mathrm{a}$ & & 0.649 & & $0.52 \mathrm{a}$ & & $37.28 a$ & & 5.55 & & 2.36 & & 7.51 & \\
\hline \multirow[t]{4}{*}{50} & & 5.17 & & $8.44 \mathrm{~b}$ & & 0.557 & & $0.577 \mathrm{~b}$ & & 0.633 & & $0.48 \mathrm{~b}$ & & $29.08 b$ & & 5.41 & & 1.97 & & 7.63 & \\
\hline & 0 & 5.27 & & $9.59 \mathrm{a}$ & & 0.641 & & $0.302 \mathrm{c}$ & & $0.714 \mathrm{a}$ & & 0.50 & & $27.32 \mathrm{~b}$ & & $5.89 \mathrm{a}$ & & 2.03 & & 7.75 & \\
\hline & 0.3 & 5.29 & & $8.51 \mathrm{~b}$ & & 0.546 & & $0.609 \mathrm{~b}$ & & $0.629 \mathrm{~b}$ & & 0.49 & & $29.49 b$ & & $4.72 \mathrm{~b}$ & & 2.22 & & 7.63 & \\
\hline & 0.9 & 5.25 & & $8.06 \mathrm{~b}$ & & 0.603 & & $0.940 \mathrm{a}$ & & $0.580 \mathrm{~b}$ & & 0.52 & & $42.74 \mathrm{a}$ & & $5.84 \mathrm{a}$ & & 2.26 & & 7.33 & \\
\hline \multirow[t]{3}{*}{100} & 0 & 5.27 & & 9.95 & & 0.638 & & $0.316 \mathrm{e}$ & & 0.734 & & 0.52 & & 29.45 & & $5.68 \mathrm{ab}$ & & 1.96 & & $8.07 \mathrm{a}$ & \\
\hline & 0.3 & 5.36 & & 8.55 & & 0.636 & & $0.648 \mathrm{c}$ & & 0.594 & & 0.52 & & 33.30 & & $5.12 \mathrm{~b}$ & & 2.38 & & $7.28 \mathrm{~b}$ & \\
\hline & 0.9 & 5.47 & & 8.50 & & 0.636 & & $1.006 \mathrm{a}$ & & 0.620 & & 0.54 & & 49.09 & & $5.85 \mathrm{ab}$ & & 2.75 & & $7.18 \mathrm{~b}$ & \\
\hline \multirow[t]{3}{*}{50} & 0 & 5.28 & & 9.22 & & 0.644 & & $0.288 \mathrm{e}$ & & 0.694 & & 0.47 & & 25.18 & & $6.11 \mathrm{a}$ & & 2.09 & & $7.42 \mathrm{ab}$ & \\
\hline & 0.3 & 5.22 & & 8.47 & & 0.456 & & $0.570 \mathrm{~d}$ & & 0.665 & & 0.46 & & 25.67 & & $4.31 \mathrm{c}$ & & 2.07 & & $7.99 \mathrm{ab}$ & \\
\hline & 0.9 & 5.02 & & 7.63 & & 0.570 & & $0.873 \mathrm{~b}$ & & 0.540 & & 0.49 & & 36.39 & & $5.82 \mathrm{ab}$ & & 1.77 & & $7.48 \mathrm{ab}$ & \\
\hline
\end{tabular}

z: Each value is the mean of 3 replications; y: numbers in the same column follwed by different letter are sugnificantly different, according to the LSD ( $p<0.05)$; v: 50 or $100 \%$ nitrogen in the nutrient solution; $\mathrm{u}: 0,0.3$ or $0.9 \%$ Amino16 in the nutrient solution; $\mathrm{s}$ : total variance; r: ${ }^{* * *},{ }^{* * *}$ significant effect at $p<0.05,0.01$ or 0.001 , respectively. 


\section{Discussion}

The consistence in plant weight of both green and red baby lettuce grown in reduced $\mathrm{N}$ implies that mineral nutrition during the production of baby-size lettuce may be lowered with consequent benefits regarding the production cost and environmental protection. In our study, the concentration of $\mathrm{N}$ in the sufficiently supplied nutrient solutions $(100 \% \mathrm{~N})$ was significantly lower than the those reported by Gunes et al. [42] and Konstantopoulou et al. [33,34] in hydroponically grown lettuce in nutrient film technique NFT or in rockwool substrate in open hydroponic systems. The reason for selecting a lower $\mathrm{N}$ concentration was the fact that plants were harvested in the baby stage before leaves expand at a maximum rate and thus the plants suffer from the high plant density used.

The use of amino acids solutions as a replacement of part of the $\mathrm{N}$ fertilization successfully sustained lettuce plant growth and yield in a soilless growth system, implying an efficient absorption of amino acids by plants' roots. Indeed, the replacement of $20 \%$ of nitrate in nutrient solutions with amino acids in soilless culture, similar to our study, did not affect plant weight of lettuce or onion [41,42], whereas fresh weight of pak choi either increased or decreased, depending on the individual amino acids source [44]. Contrary to the results of Colla et al. [56], who reported that a protein hydrolysate treatment promoted coleoptile elongation in corn and tomato root growth, by increasing the Amino16 ${ }^{\circledR}$ concentration in the nutrient solution $(0.9 \%)$, the plant growth decreased in both green and red baby lettuce. This result may be the consequence of the increased electrical conductivity (EC) of the nutrient solution after amino acids supplementation, inducing an osmotic stress to the plants. Indeed, proline content, an osmolytic substance that is synthesized in plants under stress conditions, of both green and red lettuce plants that grew on a solution supplemented with this high dosage of amino acids solution was significantly higher than that of either the control or the low amino acids solutions.

Lettuce is considered as a moderately salt-sensitive vegetable [57] and significant yield reduction has been observed in baby lettuce grown on floating trays when EC increased from 2.8 to $3.4 \mathrm{mS} / \mathrm{cm}$, without being further affected at $4.8 \mathrm{mS} / \mathrm{cm}$ [58]. In our study, however, the EC of the nutrient solution increased beyond that level and a decrease in plant weight was observed in both green and red lettuce. Moreover, root length was negatively affected even by the slight EC increase in the red lettuce, as well as in green lettuce at the extreme EC level. The highest amino acids concentration might have been perceived as a stressful condition by the plants, as also confirmed by the increased synthesis of prolines when lettuce plants were grown on the $0.9 \%$ nutrient solution. Our results are in accordance with those of Shannon and Grieve [57], who reported that high EC due to salinity reduces yield and root length of plants, but in turn may have some favorable effects on vegetable quality.

In fact, according to Chisari et al. and Scuderi et al. [59,60], fresh cut romaine lettuce benefited from increased pre-harvest salinity during growth on a floating tray system, since product respiration, decay development, and activity of polyphenoloxidase and peroxidase decreased, while color, phenolic content, and antioxidant capacity were best maintained, prolonging the postharvest life of the fresh product. Therefore, the proper EC level in a soilless cultivation should be accordingly selected in order to achieve the highest possible nutritional quality, without detrimental effect on plant weight [61].

Analysis of variance indicated that although $\mathrm{N}$ concentration $\times$ amino acids supplement interaction had a significant effect on some parameters, its relative contribution to the total variance was higher than that of the individual sources ( $\mathrm{N}$ concentration or amino acids supplement) only for soluble solids (Table 2) and $\mathrm{Cu}$ content (Table 3) of green baby lettuce and for $\mathrm{Zn}$ content of red baby lettuce (Table 6). Dry weight of pak choi was negatively affected when part of the nitrate fertilization was replaced with amino acids in the nutrient solution, but this effect was amino acid-type dependent [44]. In contrast, partial replacement of nitrate $\mathrm{N}$ with mixed amino acid solution in NFT grown lettuce plants did not affect dry matter content [41,42]. Our above results are in accordance with those of Stefanelli et al. [36], who concluded that a reduced nitrate $\mathrm{N}$ fertilization can be applied in soilless grown lettuce production without affecting crop yield, total phenol, and ascorbic acid content, while reducing nitrate content in plant tissues. Similarly, in field grown lettuce, tissue nitrate was lowered by the reduction of mineral $\mathrm{N}$ fertilization, without the plant growth rate being significantly affected $[21,30]$. This reduction of 
mineral $\mathrm{N}$ supply, however, should be performed carefully, as long it is unrealistic to eliminate all nitrate from lettuce tissue by completely banning $\mathrm{N}$ fertilizer use [3].

The effect of amino acid application on $\mathrm{NO}_{3}{ }^{-}$reduction has also been demonstrated in winter onion [41] and lettuce [42] grown in NFT. The interpretation of this effect is contradictory among researchers, with others stating that amino acids are preferably absorbed by plants as a reduced $\mathrm{N}$ source [41,42], while others claim that the main role of amino acids on nitrate uptake and assimilation is the regulation of many processes and metabolic pathways of plant $\mathrm{N}$ metabolism, such as nitrate and nitrite reductase and glutamine synthetase activities [13,40], or even the inhibition of the expression of HvNRT2 transcription in roots, which induces the synthesis of the mRNA encoding nitrate transporter that is directly related to nitrate uptake [62]. Alternatively, Aslam et al. [43] suggested that induction of the $\mathrm{NO}_{3}{ }^{-}$transporter in the presence of amino acids may be normal, but the turnover of the mRNA encoding the transporter may be increased. In addition, plant sap must have negative water potential relative to the external solution to maintain cell turgor to transport nutrients from the roots. If tissue nitrate is a major component of the regulation of water potential within plant cells, it should vary with solution EC [21].

Although, the nitrate content of lettuce grown on $100 \% \mathrm{~N}$ was much lower than the maximum limits established by the European Commission Regulation (ECR) No. 563/2002 for lettuce production under cover $(4500 \mathrm{mg} / \mathrm{kg} \mathrm{FW})$, the use of amino acids as a replacement of mineral supplemental fertilization is of high importance in order to minimize $\mathrm{NO}_{3}{ }^{-}$content in consumed vegetables. Indeed, the European Commission Regulation (ECR) No. 1881/2006 implies that the presence of contaminants must be reduced more thoroughly wherever possible by means of good agricultural practices in order to achieve a higher level of health protection, especially for sensitive groups of the population. Moreover, currently the market demand for low-size lettuces, such as the baby-size ones, is increasing [63] and, therefore, more innovation as far as agricultural practices implementation should be exploited to fulfill consumer expectations.

In conclusion, the production of high-quality baby-size lettuces grown on floating systems is feasible, if factors such as proper $\mathrm{N}$ concentration and amino acids application rate are taken into consideration based on the type of lettuce to be grown. $\mathrm{N}$ reduction in the nutrient solution of lettuce production in a floating system did not affect the yield of green and red baby lettuce, while, simultaneously, it reduced nitrates and occasionally increased dry mater, soluble solid, phenol, chlorophyll content, and antioxidant capacity. The addition of $0.3 \%$ Amino16 ${ }^{\circledR}$ in the nutrient solution of both $\mathrm{N}$ levels (100 and 50\%) did not affect the yield of lettuce, with the exception of red type, and significantly improved nutritional quality in terms of soluble solid and phenol content and antioxidant capacity, while reducing nitrate accumulation.

Author Contributions: Conceptualization, P.T. and A.S.S.; Methodology, P.T., D.S.K., and D.-C.A.; Software, P.T. and D.-C.A.; Formal Analysis, A.S.S.; Investigation, D.S.K. and D.-C.A.; Data Curation, P.T. and D.-C.A.; Writing-Original Draft Preparation, P.T., D.S.K., D.-C.A.; Writing-Review and Editing, N.B. and A.S.S.; Supervision, N.B. and A.S.S. All authors have read and agreed to the published version of the manuscript.

Funding: The publishing fee for the article was obtained from EVYP LLP.

Conflicts of Interest: The authors declare no conflict of interest.

\section{References}

1. Broadley, M.R.; Seginer, I.; Burns, A.; Escobar-Gutiérrez, A.J.; Burns, I.G.; White, P.J. The nitrogen and nitrate economy of butterhead lettuce (Lactuca sativa var. capitata L.). J. Exp. Bot. 2003, 54, 2081-2090. [CrossRef]

2. Salomez, J.; Hofman, G. Nitrogen nutrition effects on nitrate accumulation of soil-grown greenhouse butterhead lettuce. Commun. Soil Sci. Plant Anal. 2009, 40, 620-632. [CrossRef]

3. Montemurro, F. Are organic fertilizing strategies able to improve lettuce yield, use of nitrogen and $\mathrm{N}$ statues? J. Plant Nutr. 2010, 33, 1980-1997. [CrossRef]

4. Santamaria, P. Nitrate in vegetables: Toxicity, content, intake and EC regulation. J. Sci. Food Agric. 2006, 86, 10-17. [CrossRef] 
5. Liu, W.K.; Yang, Q.C.; Qiu, Z.P. Spatiotemporal changes of nitrate and Vc contents in hydroponic lettuce treated with various nitrogen-free solutions. Acta Agric. Scand. Sect. B Soil Plant Sci. 2012, 62, 286-290. [CrossRef]

6. Gonnella, M.; Serio, F.; Conversa, G.; Santamaria, P. Yield and quality of lettuce grown in floating system using different sowing density and plant spatial arrangements. Acta Hortic. 2003, 614, 687-692. [CrossRef]

7. Zanin, G.; Ponchia, G.; Sambo, P. Yield and quality of vegetables grown in a floating system for ready-to-eat produce. Acta Hortic. 2009, 807, 433-438. [CrossRef]

8. Rodríguez-Hidalgo, S.; Artés-Hernández, F.; Gómez, P.A.; Fernández, J.A.; Artés, F. Quality of fresh-cut baby spinach grown under a floating trays system as affected by nitrogen fertilisation and innovative packaging. J. Sci. Food Agric. 2010, 90, 1089-1097. [CrossRef] [PubMed]

9. Jackson, L.E.; Stivers, L.J.; Warden, B.T.; Tanji, K.K. Crop nitrogen utilization and soil nitrate loss in a lettuce field. Fertil. Sci. Res. 1994, 37, 93-105. [CrossRef]

10. Owen, A.G.; Jones, D.L. Competition for amino acids between wheat roots and rhizosphere microorganisms and the role of amino acids in plant $\mathrm{N}$ acquisition. Soil Biol. Biochem. 2001, 33, 651-657. [CrossRef]

11. Blom-zandstra, M. Nitrate accumulation in vegetables and its relationship to quality. Ann. Appl. Biol. 1989, 115, 553-561. [CrossRef]

12. Vernieri, P.; Borghesi, E.; Ferrante, A.; Magnani, G. Application of biostimulants in floating system for improving rocket quality. J. Food Agric. Environ. 2005, 33, 86-88.

13. Liu, X.-Q.; Ko, K.-Y.; Kim, S.-H.; Lee, K. Effect of amino acid fertilization on nitrate assimilation of leafy radish and soil chemical properties in high nitrate soil. Commun. Soil Sci. Plant Anal. 2008, 39, 269-281. [CrossRef]

14. Padgett, P.E.; Leonard, R.T. Regulation of nitrate uptake by amino acids in maize cell suspension culture and intact roots. Plant Soil 1993, 155, 159-161. [CrossRef]

15. Pôrto, M.L.; Alves, J.D.C.; De Souza, A.P.; Araújo, R.D.C.; De Arruda, J.A. Nitrate production and accumulation in lettuce as affected by mineral nitrogen supply and organic fertilization. Hortic. Bras. 2008, 26, 227-230. [CrossRef]

16. Maynard, D.N.; Barker, A.V.; Minotti, P.L.; Peck, N.H. Nitrate accumulation in vegetables. In Advances in Agronomy; Brady, N.C., Ed.; Academic Press Inc.: Cambridge, MA, USA, 1976; pp. 71-118.

17. Santamaria, P.; Elia, A.; Gonnella, M. Changes in nitrate accumulation and growth of endive plants during light period as affected by nitrogen level and form 1. J. Plant Nutr. 1997, 20, 1255-1266. [CrossRef]

18. Wang, Z.-H.; Li, S.-X.; Malhi, S. Effects of fertilization and other agronomic measures on nutritional quality of crops. J. Sci. Food Agric. 2008, 88, 7-23. [CrossRef]

19. Elwan, M.W.M.; Abd El-Hamed, K.E. Influence of nitrogen form, growing season and sulfur fertilization on yield and the content of nitrate and vitamin C of broccoli. Sci. Hortic. 2011, 127, 181-187. [CrossRef]

20. Colla, G.; Kim, H.-J.; Kyriacou, M.C.; Rouphael, Y. Nitrate in fruits and vegetables. Sci. Hortic. 2018, 237, 221-238. [CrossRef]

21. Gent, M.P.N. Solution electrical conductivity and ratio of nitrate to other nutrients affect accumulation of nitrate in hydroponic lettuce. HortScience 2003, 38, 222-227. [CrossRef]

22. Burns, I.G.; Zhang, K.; Turner, M.K.; Meacham, M.; Al-Redhiman, K.; Lynn, J.; Broadley, M.R.; Hand, P.; Pink, D. Screening for genotype and environment effects on nitrate accumulation in 24 species of young lettuce. J. Sci. Food Agric. 2011, 91, 553-562. [CrossRef] [PubMed]

23. Escobar-Gutiérrez, A.J.; Burns, I.G.; Lee, A.; Edmondson, R.N. Screening lettuce cultivars for low nitrate content during summer and winter production. J. Hortic. Sci. Biotechnol. 2002, 77, 232-237. [CrossRef]

24. Burns, I.G.; Durnford, J.; Lynn, J.; Mcclement, S.; Hand, P.; Pink, D. The influence of genetic variation and nitrogen source on nitrate accumulation and iso-osmotic regulation by lettuce. Plant Soil 2012, 352, 321-339. [CrossRef]

25. Eppendorfer, W.H. Free and total amino acid composition of edible parts of beans, kale, spinach, cauliflower and potatoes as influenced by nitrogen fertilisation and phosphorus and potassium deficiency. J. Sci. Food Agric. 1996, 71, 449-458. [CrossRef]

26. Fontes, P.C.R.; Pereira, P.R.G.; Conde, R.M. Critical chlorophyll, total nitrogen, and nitrate-nitrogen in leaves associated to maximum lettuce yield. J. Plant Nutr. 1997, 20, 1061-1068. [CrossRef]

27. Abu-Rayyan, A.; Kharawish, B.H.; Al-Ismail, K. Nitrate content in lettuce (Lactuca sativa L) heads in relation to plant spacing, nitrogen form and irrigation level. J. Sci. Food Agric. 2004, 84, 931-936. [CrossRef] 
28. Burns Ian, G.; Kefeng, Z.; Mary, K.T.; James, L.; Sandy, M.C.; Paul, H.; David, P. Genotype and environment effects on nitrate accumulation in a diversity set of lettuce accessions at commercial maturity: The influence of nitrate uptake and assimilation, osmotic interactions and shoot weight and development. J. Sci. Food Agric. 2011, 91, 2217-2233. [CrossRef]

29. Ferentinos, K.P.; Albright, L.D.; Ramani, D.V. Optimal light integral and carbon dioxide concentration combinations for lettuce in ventilated greenhouses. J. Agric. Eng. Res. 2000, 77, 309-315. [CrossRef]

30. Tsouvaltzis, P.; Koukounaras, A.; Siomos, A.S. Application of amino acids improves lettuce crop uniformity and inhibits nitrate accumulation induced by the supplemental inorganic nitrogen fertilization. Int. J. Agric. Biol. 2014, 16, 951-955.

31. Santamaria, P.; Elia, A. Producing nitrate-free endive heads: Effect of nitrogen form on growth, yield, and ion composition of endive. J. Am. Soc. Hortic. Sci. 1997, 122, 140-145. [CrossRef]

32. Quattrini, E.; Penati, M.; Alberici, A.; Martinetti, L.; Marino Gallina, P.; Ferrante, A.; Schiavi, M. Effect of the reduction of nutrient solution concentration on leafy vegetables quality grown in floating system. Acta Hortic. 2008, 801, 1167-1176.

33. Konstantopoulou, E.; Kapotis, G.; Salachas, G.; Petropoulos, S.A.; Karapanos, I.C.; Passam, H.C. Nutritional quality of greenhouse lettuce at harvest and after storage in relation to $\mathrm{N}$ application and cultivation season. Sci. Hortic. 2010, 125, 93.e1-93.e5. [CrossRef]

34. Konstantopoulou, E.; Kapotis, G.; Salachas, G.; Petropoulos, S.A.; Chatzieustratiou, E.; Karapanos, I.C.; Passam, H.C. Effect of nitrogen application on growth parameters, yield and leaf nitrate content of greenhouse lettuce cultivated during three seasons. J. Plant Nutr. 2012, 35, 1246-1254. [CrossRef]

35. Gent, M.P.N. Rate of change of composition of lettuce in response to nitrogen depletion or re-supply. J. Sci. Food Agric. 2012, 92, 3007-3015. [CrossRef]

36. Stefanelli, D.; Brady, S.; Winkler, S.; Jones, R.B.; Tomkins, B.T. Lettuce (Lactuca sativa L.) growth and quality response to applied nitrogen under hydroponic conditions. Acta Hortic. 2012, 353-359. [CrossRef]

37. Fischer, W.N.; André, B.; Rentsch, D.; Krolkiewicz, S.; Tegeder, M.; Breitkreuz, K.; Frommer, W.B. Amino acid transport in plants. Trends Plant Sci. 1998, 3, 188-195. [CrossRef]

38. El-Naggar, A.; El-Araby, A.; De Neergaard, A.; Høgh-Jensen, H. Crop responses to 15N-labelled organic and inorganic nitrogen sources. Nutr. Cycl. Agroecosyst. 2008, 80, 49-60. [CrossRef]

39. El-Naggar, A.; De Neergaard, A.; El-Araby, A.; Høgh-Jensen, H. Simultaneous uptake of multiple amino acids by Wheat. J. Plant Nutr. 2009, 32, 725-740. [CrossRef]

40. Liu, X.; Chen, H.; Ni, Q.; Seung, L.K. Evaluation of the role of mixed amino acids in nitrate uptake andassimilation in leafy radish by using $15 \mathrm{~N}$-labeled nitrate. Agric. Sci. China 2008, 7, 1196-1202. [CrossRef]

41. Güneş, A.; İnal, A.; Aktaş, M. Reducing nitrate content of NFT grown winter onion plants (Allium cepa L.) by partial replacement of $\mathrm{NO}_{3}$ with amino acid in nutrient solution. Sci. Hortic. 1996, 65, 203-208. [CrossRef]

42. Gunes, A.; Post, W.H.K.; Kirkby, E.A.; Aktas, M. Influence of partial replacement on nitrate by amino acid nitrogen or urea in the nutrient medium on nitrate accumulation in NFT grown winter lettuce. J. Plant Nutr. 1994, 17, 1929-1938. [CrossRef]

43. Aslam, M.; Travis, R.L.; Rains, D.W. Differential effect of amino acids on nitrate uptake and reduction systems in barley roots. Plant Sci. 2001, 160, 219-228. [CrossRef]

44. Wang, H.-J.; Wu, L.-H.; Wang, M.-Y.; Zhu, Y.-H.; Tao, Q.-N.; Zhang, F.-S. Effects of amino acids replacing nitrate on growth, nitrate accumulation, and macroelement concentrations in pak-choi (Brassica chinensis L.). Pedosphere 2007, 17, 595-600. [CrossRef]

45. Du Jardin, P. Plant biostimulants: Definition, concept, main categories and regulation. Sci. Hortic. 2015, 196, 3-14. [CrossRef]

46. Rouphael, Y.; Colla, G. Editorial: Biostimulants in Agriculture. Front. Plant Sci. 2020, 11, 1-7. [CrossRef]

47. European Patent Office. European Patent Application No. 11386012.6. of Aminoacids of Plant Origin and Method of Production. Patent No. EP 2537823 A1, 26 December 2012.

48. Cataldo, D.A.; Maroon, M.; Schrader, L.E.; Youngs, V.L. Rapid colorimetric determination of nitrate in plant tissue by nitration of salicylic acid. Commun. Soil Sci. Plant Anal. 1975, 6, 71-80. [CrossRef]

49. Scalbert, A.; Monties, B.; Janin, G. Tannins in wood: Comparison of different estimation methods. J. Agric. Food Chem. 1989, 37, 1324-1329. [CrossRef]

50. Brand-Williams, W.; Cuvelier, M.E.; Berset, C. Use of a free radical method to evaluate antioxidant activity. LWT Food Sci. Technol. 1995, 28, 25-30. [CrossRef] 
51. Arnon, D.I. Copper enzymes in isolated chloroplasts. Polyphenoloxidase in Beta vulgaris. Plant Physiol. 1949, 24, 1-15. [CrossRef]

52. Fuleki, T.; Francis, F.J. Quantitative methods for anthocyanins. 1. Extraction and Determination of Total Anthocyanin in Cranberries. J. Food Sci. 1968, 33, 72-77. [CrossRef]

53. Troll, W.; Lindsley, J. A photometric method for the determination of proline. J. Biol. Chem. 1955, 215, 655-660. [PubMed]

54. Bremner, J.M. Nitrogen-Total. In Methods of Soil Analysis Part 3 Chemical Methods; Helmke, P.A., Loeppert, R.H., Eds.; SSSA Book Ser 53; SSSA: Madison, WI, USA; ASA: Schaumburg, IL, USA, 1996; pp. 1085-1122.

55. Olsen, S.R.; Sommers, L.E. Phosphorus. Methods of Soil Analysis Part 2 Chemical and Microbiological Properties-Agronomy Monograph No. 9, 2nd ed.; Page, A.L., Ed.; ASA: Schaumburg, IL, USA; SSSA: Madison, WI, USA, 1982; pp. 403-430.

56. Colla, G.; Rouphael, Y.; Canaguier, R.; Svecova, E.; Cardarelli, M. Biostimulant action of a plant-derived protein hydrolysate produced through enzymatic hydrolysis. Front. Plant Sci. 2014, 5, 1-6. [CrossRef] [PubMed]

57. Shannon, M.C.; Grieve, C.M. Tolerance of vegetable crops to salinity. Sci. Hortic. 1999, 78, 5-38. [CrossRef]

58. Scuderi, D.; Giuffrida, F.; Noto, G. Effects of nutrient solution EC on yield, quality andshelf-life of lettuce grown in floating system. Acta Hortic. 2009, 807, 221-226. [CrossRef]

59. Chisari, M.; Todaro, A.; Barbagallo, R.N.; Spagna, G. Salinity effects on enzymatic browning and antioxidant capacity of fresh-cut baby Romaine lettuce (Lactuca sativa L. cv. Duende). Food Chem. 2010, 119, 1502-1506. [CrossRef]

60. Scuderi, D.; Restuccia, C.; Chisari, M.; Barbagallo, R.N.; Caggia, C.; Giuffrida, F. Salinity of nutrient solution influences the shelf-life of fresh-cut lettuce grown in floating system. Postharvest Biol. Technol. 2011, 59, 132-137. [CrossRef]

61. Bonasia, A.; Lazzizera, C.; Elia, A.; Conversa, G. Nutritional, biophysical and physiological characteristics of wild rocket genotypes as affected by soilless cultivation system, salinity level of nutrient solution and growing period. Front. Plant Sci. 2017, 8, 300. [CrossRef]

62. Vidmar, J.J.; Zhuo, D.; Siddiqi, M.Y.; Schjoerring, J.K.; Touraine, B.; Glass, A.D.M. Regulation of high-affinity nitrate transporter genes and high-affinity nitrateinflux by nitrogen pools in roots of barley. Plant Physiol. 2000, 123, 307-318. [CrossRef]

63. Martínez-Sánchez, A.; Luna, M.C.; Selma, M.V.; Tudela, J.A.; Abad, J.; Gil, M.I. Baby-leaf and multi-leaf of green and red lettuces are suitable raw materials for the fresh-cut industry. Postharvest Biol. Technol. 2012, 63, 1-10. [CrossRef]

(C) 2020 by the authors. Licensee MDPI, Basel, Switzerland. This article is an open access article distributed under the terms and conditions of the Creative Commons Attribution (CC BY) license (http://creativecommons.org/licenses/by/4.0/). 